\title{
ON THE CHAIN RULE FORMULAS FOR DIVERGENCES AND APPLICATIONS TO CONSERVATION LAWS
}

\author{
On the occasion of the 60th birthday of Nicola Fusco
}

GRAZIANO CRASTA AND VIRGINIA DE CICCO

\begin{abstract}
In this paper we prove a nonautonomous chain rule formula for the distributional divergence of the composite function $\mathbf{v}(x)=\boldsymbol{B}(x, u(x))$, where $\boldsymbol{B}(\cdot, t)$ is a divergence-measure vector field and $u$ is a function of bounded variation. As an application, we prove a uniqueness result for scalar conservation laws with discontinuous flux.
\end{abstract}

\section{INTRODUCTION}

Nonautonomous chain rules formulas in $B V$ have been successfully used in the study of semicontinuity properties of integral functionals (see $[12,14,15,16]$ ) and conservation laws with discontinuous flux of the form

$$
u_{t}+\operatorname{div} \boldsymbol{B}(x, u)=0, \quad(t, x) \in(0,+\infty) \times \mathbb{R}^{N}
$$

(see $[9,10,11]$ and also $[21,22]$ in the autonomous case). In this paper we shall restrict our attention only to this second kind of application.

In order to clarify the connection between chain rule formulas and uniqueness results for the Cauchy problems associated with (1), it will be convenient to recall some previous results.

In [10] the authors considered a flux $\boldsymbol{B}$ such that $\boldsymbol{B}(\cdot, z)$ is a special function of bounded variation (SBV) and of class $C^{1}$ with respect to the second variable. A uniqueness result for (1) is then obtained in the class of $B V$ functions by using the chain rule formula proven in [1] for the composite function $\mathbf{v}(x):=\boldsymbol{B}(x, u(x)$ ). (For the sake of completeness we recall that, under the same structural hypotheses on the flux, a similar uniqueness result has been recently obtained in [11] for weak entropy solutions, without the $B V$ regularity requirement.)

On the other hand, Panov proved in [19] an existence result of entropy solutions in the case of discontinuous fluxes $\boldsymbol{B}(x, z)$ such that $\boldsymbol{B}(\cdot, z)$ is a vector field whose distributional divergence $\operatorname{div}_{x} \boldsymbol{B}(\cdot, z)$ is a measure (see $[6,7,8]$ for a general theory of bounded divergencemeasure vector field). This assumption on $\operatorname{div}_{x} \boldsymbol{B}(\cdot, z)$, rather than requiring $\boldsymbol{B}(\cdot, z) \in$ $S B V$, is indeed natural when looking for entropy solutions of (1).

The structure of the proof of the uniqueness result in [10] can be adapted to this more general situation, provided that one can prove a suitable chain rule formula. This is exactly the aim of this paper: in Section 4 we shall prove a nonautonomous chain rule formula for the divergence of the vector field $\mathbf{v}(x):=\boldsymbol{B}(x, u(x))$, where $\boldsymbol{B}(\cdot, t)$ is a divergence-measure

Date: May 31, 2016.

2010 Mathematics Subject Classification. 26A45,35L65.

Key words and phrases. Chain rule, divergence. 
vector field, of class $C^{1}$ with respect to the second variable, and $u: \mathbb{R}^{N} \rightarrow \mathbb{R}$ is a function of bounded variation. Then, we can mimic the proof in [10] in order to obtain, under these assumptions on $\boldsymbol{B}$, a uniqueness result for $B V$ solutions of the Cauchy problems associated with (1) (see Section 5). We stress that this is not a genuine well-posedness result, since uniqueness of solutions has been proven in a class of functions which is smaller than the one for which existence has been obtained by Panov.

Before stating our results in a more precise way, let us recall the state of the art about chain rule formulas, starting from the autonomous (i.e., independent of $x$ ) case.

The first result concerning distributional derivatives is the one proved by Vol'pert in [21] (see also [22]), in view of applications to the study of quasilinear hyperbolic equations. He established a chain rule formula for distributional derivatives of the composite function $v(x)=B(u(x))$, where $u: \Omega \rightarrow \mathbb{R}$ has bounded variation in the open subset $\Omega$ of $\mathbb{R}^{N}$ and $B: \mathbb{R} \rightarrow \mathbb{R}$ is continuously differentiable. He proved that $v$ has bounded variation and its distributional derivative $D v$ (which is a Radon measure on $\Omega$ ) admits an explicit representation in terms of the classical derivative $B^{\prime}$ and of the distributional derivative $D u$. More precisely, the following equality holds

$$
D v=B^{\prime}(u) \nabla u \mathcal{L}^{N}+B^{\prime}(\widetilde{u}) D^{c} u+\left[B\left(u^{+}\right)-B\left(u^{-}\right)\right] \nu_{u} \mathcal{H}^{N-1}\left\llcorner J_{u},\right.
$$

in the sense of measures, where

$$
D u=\nabla u \mathcal{L}^{N}+D^{c} u+\left(u^{+}-u^{-}\right) \nu_{u} \mathcal{H}^{N-1}\left\llcorner J_{u}\right.
$$

is the decomposition of $D u$ into its absolutely continuous part $\nabla u \mathcal{L}^{N}$ with respect to the Lebesgue measure $\mathcal{L}^{N}$, its Cantor part $D^{c} u$ and its jump part, which in turn is a measure concentrated on the $\mathcal{H}^{N-1}$-rectifiable jump set $J_{u}$ of $u$. Here, $\nu_{u}$ denotes the measure theoretical unit normal to $J_{u}, \widetilde{u}$ is the approximate limit of $u$ and $u^{+}, u^{-}$are the traces of $u$ on $J_{u}$. (Here and in the following we refer to Chapter 3 of [5] for notations and the basic facts concerning $B V$ functions.)

An identity similar to (2) holds also in the vectorial case (see Theorem 3.96 in [5]), namely when $\mathbf{u}: \mathbb{R}^{N} \rightarrow \mathbb{R}^{h}$ has bounded variation and $B: \mathbb{R}^{h} \rightarrow \mathbb{R}$ is continuously differentiable. In this case, (2) can be written as

$$
D v=\nabla B(\mathbf{u}) \nabla \mathbf{u} \mathcal{L}^{N}+\nabla B(\widetilde{\mathbf{u}}) D^{c} \mathbf{u}+\left[B\left(\mathbf{u}^{+}\right)-B\left(\mathbf{u}^{-}\right)\right] \nu_{\mathbf{u}} \mathcal{H}^{N-1}\left\llcorner J_{\mathbf{u}} .\right.
$$

A further extension, that we are not going to use in the present paper, concerns the case when $B$ is only a Lipschitz continuous function. In this case, a general form of the formula was proved by Ambrosio and Dal Maso in [3] (see also [18], Theorem 3.99 in [5] for the scalar case and [13] for the nonautonomous case).

Recently, analogous chain rule formulas have been obtained in the case of an explicit dependence with respect to the space variables $x$, especially in view of applications to semicontinuity results for convex integral nonautonomous functionals (see $[12,14,15,16]$ ) and to conservation laws with discontinuous flux (see $[9,10,11]$ ). This amounts to describe the distributional derivative of the composite function $\mathbf{v}(x)=\boldsymbol{B}(x, \mathbf{u}(x))$, where $\boldsymbol{B}(x, \cdot)$ is continuously differentiable and $\boldsymbol{B}(\cdot, \mathbf{z})$ and $\mathbf{u}$ are functions with low regularity (which will be specified later). These formulas contain another derivation term due to the presence of the explicit dependence on $x$.

In the case of $\mathbf{u}$ and $\boldsymbol{B}$ regular functions, the classical chain rule formula

$$
\nabla \mathbf{v}(x)=\nabla_{x} \boldsymbol{B}(x, \mathbf{u}(x))+\nabla_{\mathbf{z}} \boldsymbol{B}(x, \mathbf{u}(x)) \cdot \nabla \mathbf{u}(x), \quad x \in \mathbb{R}^{N},
$$

is a pointwise identity and the derivatives here occurring are the classical ones. 
Clearly, when $\boldsymbol{B}(\cdot, \mathbf{z})$ or $\mathbf{u}$ (or both) are not regular functions, then also $\mathbf{v}$ need not be regular and a number of extra terms will appear, as in the previous formulas (2) and (3).

In the main result of the paper, stated in Theorem 4.7, we assume that $h=1$, the function $\boldsymbol{B}(x, \cdot)$ is $C^{1}, \operatorname{div}_{x} \boldsymbol{B}(\cdot, z)$ is a Radon measure, and $u$ is a scalar function of bounded variation. As we shall see, in order to obtain the formula in such generality, we need to assume a-priori the existence of the strong traces $\boldsymbol{B}^{ \pm}(\cdot, z)$ of $\boldsymbol{B}(\cdot, z)$ on a $\mathcal{H}^{N-1}$-rectifiable universal singular set $\mathcal{N}$, independent of $z$ (see Section 4 ). Nevertheless, we think that this restriction should not be too much severe in view of applications to conservation laws with discontinuous flux (see Section 5 and Remark 5.2). Clearly, in this case one can expect a chain rule formula only for the divergence of the composite function $\mathbf{v}(x)=\boldsymbol{B}(x, u(x)))$. Namely, we shall prove that the distributional divergence of $\mathbf{v}$ is given by

$$
\begin{aligned}
\operatorname{div} \mathbf{v}(x)) & =\left.\operatorname{div}_{x} \boldsymbol{B}(x, t)\right|_{t=u(x)} \\
& +\langle\boldsymbol{b}(x, \widetilde{u}(x)), \widetilde{D} u\rangle+\left\langle\boldsymbol{B}^{*}\left(x, u^{+}\right)-\boldsymbol{B}^{*}\left(x, u^{-}\right), \nu_{u}\right\rangle \mathcal{H}^{N-1}\left\llcorner\mathcal{N} \cup J_{u}\right.
\end{aligned}
$$

in the sense of measure, where $\boldsymbol{B}^{*}(x, z)=\frac{1}{2}\left[\boldsymbol{B}^{+}(x, z)+\boldsymbol{B}^{-}(x, z)\right]$ and the measure $\operatorname{div}_{x} \boldsymbol{B}(x, z)$, depending on the parameter $z$, is computed in $z=u(x)$ in a suitable sense (see Remark 4.10). The proof is based on the regularization argument used in [15].

We shall see that, when $u$ is a function of bounded variation, this explicit formula for div $\mathbf{v}$ can be used to obtain uniqueness results for (1). This improves the analogous uniqueness result previously obtained in [10], using the chain rule formula for $\mathbf{v}$, assuming $\boldsymbol{B}(\cdot, z)$ a special function of bounded variation. We emphasize that, interestingly enough, the proof of the uniqueness result of [10] can be retraced with minor modifications in this new setting, since what is really needed is only the chain rule formula for $\operatorname{div} \mathbf{v}$.

The structure of the paper is the following. First of all, in Sections 2 and 3 we review some known chain rule formulas that we believe will help the reader understanding the more general formula of Section 4.

More precisely, in Section 2 we recall the results proved in the case $\boldsymbol{B}(\cdot, \mathbf{z})$ not regular, but $\mathbf{u}$ regular enough (i.e., in the Sobolev space $W^{1,1}$ ). These results have been proved in [16] and [15], assuming that $\operatorname{div}_{x} \boldsymbol{B}(\cdot, \mathbf{z})$ belongs to $L^{1}$ or to the space of Radon measures respectively.

In Section 3 we review the chain rule formulas in the case when both $\boldsymbol{B}(\cdot, \mathbf{z})$ and $\mathbf{u}$ are of bounded variation, recalling the results proved in [15] and [1] in the case of scalar and vector functions respectively.

In Section 4 we prove the main result of the paper, stated in Theorem 4.7, concerning the distributional divergence of the composite function $\mathbf{v}(x)=\boldsymbol{B}(x, u(x))$.

Finally, in Section 5 we consider some applications of Theorem 4.7 to the uniqueness issue for the Cauchy problems for multidimensional scalar conservation laws with discontinuous flux.

\section{Nonautonomous Chain RULES For $u \in W^{1,1}$}

As we have already said in the Introduction, this section and the next one are devoted to the review of some known chain rule formulas.

In the following, $\Omega$ will always denote a nonempty open subset of $\mathbb{R}^{N}$.

2.1. Vectorial case $\mathbf{u} \in W^{1,1}\left(\Omega ; \mathbb{R}^{h}\right)$. The first formula of this type is established in [16] for functions $u \in W^{1,1}\left(\mathbb{R}^{N} ; \mathbb{R}^{h}\right)$ by assuming that, for every $\mathbf{z} \in \mathbb{R}^{h}, \boldsymbol{B}(\cdot, \mathbf{z})$ is an 
$L^{1}$ function whose distributional divergence belongs to $L^{1}$. (In particular, this condition holds if $\boldsymbol{B}(\cdot, \mathbf{z}) \in W^{1,1}\left(\mathbb{R}^{N} ; \mathbb{R}^{h}\right)$.)

Let us consider the space

$$
L^{1}(\operatorname{div} ; \Omega)=\left\{\mathbf{u} \in L^{1}\left(\Omega ; \mathbb{R}^{N}\right): \operatorname{div} \mathbf{u} \in L^{1}(\Omega)\right\},
$$

where div $\mathbf{u}$ denotes the distributional divergence of $\mathbf{u}$.

We recall that an $\mathcal{H}^{N-1}$-measurable set $A \subset \mathbb{R}^{N}$ is said to be countably $\mathcal{H}^{N-1}$ rectifiable if it can be covered, up to a set of vanishing $\mathcal{H}^{N-1}$ measure, by a sequence of $C^{1}$ hypersurfaces. The set $A$ is said to be purely $(N-1)$-unrectifiable if $\mathcal{H}^{N-1}(A \cap \Gamma)=0$ whenever $\Gamma$ is countably $\mathcal{H}^{N-1}$-rectifiable.

Theorem 2.1 ( $L^{1}(\operatorname{div} ; \Omega)$-dependence). Let $\boldsymbol{B}: \Omega \times \mathbb{R}^{h} \rightarrow \mathbb{R}^{N}$ be a Borel function. Assume that there exist an $\mathcal{L}^{N}$-null set $\mathcal{N}_{0} \subset \Omega$ and a purely $\mathcal{H}^{1}$-unrectifiable set $\mathcal{M} \subset \mathbb{R}^{h}$ such that

(i) for all $\mathbf{z} \in \mathbb{R}^{h}$ the function $\boldsymbol{B}(\cdot, \mathbf{z}) \in L_{\text {loc }}^{1}(\operatorname{div} ; \Omega)$;

(ii) for all $x \in \Omega \backslash \mathcal{N}_{0}$ the function $\operatorname{div}_{x} \boldsymbol{B}(x, \cdot)$ is approximately continuous in $\mathbb{R}^{h}$;

(iii) for all $x \in \Omega \backslash \mathcal{N}_{0}$ the function $\boldsymbol{B}(x, \cdot)$ is differentiable in $\mathbb{R}^{h} \backslash \mathcal{M}$ and approximately continuous in $\mathcal{M}$;

(iv) for every $\Omega^{\prime} \times D \subset \subset \Omega \times \mathbb{R}^{h}$ there exist $g \in L^{1}(\Omega)$ and $L>0$ such that

for $\mathcal{L}^{N}$-a.e. $x \in \Omega^{\prime}$ and for all $\mathbf{z} \in D$, and

$$
|\boldsymbol{B}(x, \mathbf{z})|+\left|\operatorname{div}_{x} \boldsymbol{B}(x, \mathbf{z})\right| \leq g(x)
$$

$$
\left|\nabla_{\mathbf{z}} \boldsymbol{B}(x, \mathbf{z})\right| \leq L
$$

for $\mathcal{L}^{N}$-a.e. $x \in \Omega^{\prime}$ and for all $\mathbf{z} \in D \backslash \mathcal{M}$.

Then for every $\mathbf{u} \in W^{1,1}\left(\Omega ; \mathbb{R}^{h}\right) \cap L_{\text {loc }}^{\infty}\left(\Omega ; \mathbb{R}^{h}\right)$ the function $\mathbf{v}: \Omega \rightarrow \mathbb{R}^{N}$, defined by

$$
\mathbf{v}(x)=\boldsymbol{B}(x, \mathbf{u}(x)) \quad x \in \Omega,
$$

belongs to $L_{\text {loc }}^{1}(\operatorname{div} ; \Omega)$ and

$$
\operatorname{div} \mathbf{v}(x)=\operatorname{div}_{x} \boldsymbol{B}(x, \mathbf{u}(x))+\operatorname{tr}\left(\nabla_{\mathbf{z}} \boldsymbol{B}(x, \mathbf{u}(x)) \nabla \mathbf{u}(x)\right)
$$

for $\mathcal{L}^{N}$-a.e. $x \in \Omega$, provided $\nabla_{\mathbf{z}} \boldsymbol{B}(x, \mathbf{u}(x)) \nabla \mathbf{u}(x)$ is interpreted to be zero whenever $\nabla \mathbf{u}(x)=0$, irrespective of whether $\nabla_{\mathbf{z}} \boldsymbol{B}(x, \mathbf{u}(x))$ is defined.

2.2. Scalar case $u \in W^{1,1}(\Omega)$. An important special case is given when $\boldsymbol{B}$ has the form

$$
\boldsymbol{B}(x, u)=\int_{0}^{u} \boldsymbol{b}(x, s) d s,
$$

where $\boldsymbol{b}: \Omega \times \mathbb{R} \rightarrow \mathbb{R}^{N}$. Clearly, when $\boldsymbol{B}$ is of class $C^{1}$ with respect to the second variable, it is not a real restriction assuming that it is of the form (5) for some vector field $\boldsymbol{b}$ which is continuous with respect to the same variable. On the other hand, some assumptions in Theorems 2.2 and 3.1 below can be stated in a more polished way in terms of $\boldsymbol{b}$.

In this case a formula has been established in [15] (see Theorem 3.4) for scalar functions $u \in W^{1,1}(\Omega)$ by assuming that, for every $t \in \mathbb{R}, \boldsymbol{B}(\cdot, t)$ is an $L_{\text {loc }}^{\infty}$ function whose distributional divergence is a Radon measure.

In the following, we shall denote by $\mathcal{D M}^{\infty}(\Omega)$ the space of all vector fields belonging to $L^{\infty}\left(\Omega ; \mathbb{R}^{N}\right)$ whose divergence in the sense of distribution is a Radon measure with finite total variation.

Theorem 2.2 (DM $\mathcal{M}^{\infty}$-dependence). Let $\boldsymbol{b}: \Omega \times \mathbb{R} \rightarrow \mathbb{R}^{N}$ be a locally bounded Borel function. Assume that 
(i) for $\mathcal{L}^{N}$-a.e. $x \in \Omega$ the function $\boldsymbol{b}(x, \cdot)$ is continuous in $\mathbb{R}$;

(ii) for $\mathcal{L}^{1}$-a.e. $t \in \mathbb{R}$ the function $\boldsymbol{b}(\cdot, t)$ belongs to $\mathcal{D} \mathcal{M}^{\infty}(\Omega)$;

(iii) for any compact set $H \subset \mathbb{R}$,

$$
\int_{H}\left|\operatorname{div}_{x} \boldsymbol{b}(\cdot, t)\right|(\Omega) d t<+\infty
$$

Then, for every $u \in W^{1,1}(\Omega) \cap L^{\infty}(\Omega)$, the function $\mathbf{v}: \Omega \rightarrow \mathbb{R}^{N}$, defined by

$$
\mathbf{v}(x):=\boldsymbol{B}(x, u(x))=\int_{0}^{u(x)} \boldsymbol{b}(x, t) d t
$$

belongs to $\mathcal{D M}^{\infty}(\Omega)$ and for any $\phi \in C_{0}^{1}(\Omega)$ we have

$$
\begin{aligned}
\int_{\Omega}\langle\nabla \phi(x), \mathbf{v}(x)\rangle d x= & -\int_{-\infty}^{+\infty} d t \int_{\Omega} \operatorname{sgn}(t) \chi_{\Omega_{u, t}}^{*} \phi(x) d \operatorname{div}_{x} \boldsymbol{b}(x, t) \\
& -\int_{\Omega} \phi(x)\langle\boldsymbol{b}(x, u(x)), \nabla u(x)\rangle d x
\end{aligned}
$$

where

$$
\Omega_{u, t}:=\{x \in \Omega: t \text { belongs to the segment of endpoints } 0 \text { and } u(x)\}
$$

and for a.e. $t$ the function $\chi_{\Omega_{u, t}}^{*}$ is the precise representative of the $B V$ function $\chi_{\Omega_{u, t}}$.

\section{Nonautonomous chain Rule for $u \in B V$}

3.1. The scalar case $u \in B V\left(\mathbb{R}^{N}\right)$. The case of a scalar function $u \in B V\left(\mathbb{R}^{N}\right)$ is studied in the papers [14] and [15], where it is considered again in the case $B=B(x, u)$ of the form (5). In the first paper the authors have established the validity of the chain rule by requiring a $W^{1,1}$ dependence with respect to the variable $x$, while in the second one it is assumed only a $B V$ dependence with respect to the variable $x$.

Theorem 3.1 (BV-dependence, see [15]). Let $b: \Omega \times \mathbb{R} \rightarrow \mathbb{R}$ be a locally bounded Borel function. Assume that

(i) for $\mathcal{L}^{1}$-a.e. $t \in \mathbb{R}$ the function $b(\cdot, t) \in B V(\Omega)$;

(ii) for any compact set $H \subset \mathbb{R}$,

$$
\int_{H}\left|D_{x} b(\cdot, t)\right|(\Omega) d t<+\infty .
$$

Then, for every $u \in B V(\Omega) \cap L_{\text {loc }}^{\infty}(\Omega)$, the function $v: \Omega \rightarrow \mathbb{R}$, defined by

$$
v(x):=\int_{0}^{u(x)} b(x, t) d t
$$

belongs to $B V_{\mathrm{loc}}(\Omega)$ and for any $\phi \in C_{0}^{1}(\Omega)$ we have

$$
\begin{aligned}
\int_{\Omega} \nabla \phi(x) v(x) d x= & -\int_{-\infty}^{+\infty} d t \int_{\Omega} \operatorname{sgn}(t) \chi_{\Omega_{u, t}}^{*}(x) \phi(x) d D_{x} b(x, t) \\
& -\int_{\Omega} \phi(x) b^{*}(x, \widetilde{u}(x)) \nabla u(x) d x-\int_{\Omega} \phi(x) b^{*}(x, \widetilde{u}(x)) d D^{c} u(x) \\
& -\int_{J_{u}} \phi(x) \nu_{u}(x) d \mathcal{H}^{N-1}(x) \int_{u^{-}(x)}^{u^{+}(x)} b^{*}(x, t) d t,
\end{aligned}
$$


where $\Omega_{u, t}$ is the set defined in (6), $J_{u}$ is the jump set of $u$, and $\chi_{\Omega_{u, t}}^{*}$ and $b^{*}(\cdot, t)$ are, respectively, the precise representatives of $\chi_{\Omega_{u, t}}$ and $b(\cdot, t)$.

Notice that if $b(x, t) \equiv b(t)$, then (7) reduces to the well known chain rule formula for the composition of $B V$ functions with a Lipschitz function, while, in the special case that $b(x, t) \equiv b(x),(7)$ gives the formula for the derivative of the product of two $B V$ functions.

3.2. The vectorial case $\mathbf{u} \in B V_{\text {loc }}\left(\mathbb{R}^{N} ; \mathbb{R}^{h}\right)$. More recently, a very general formula has been proven in [1] (see also [9] for $N=1$ ) for vector functions $\mathbf{u} \in B V\left(\mathbb{R}^{N}, \mathbb{R}^{h}\right.$ ).

Here the function $B: \mathbb{R}^{N} \times \mathbb{R}^{h} \rightarrow \mathbb{R}$ is required to satisfy the following assumptions:

(a) $x \mapsto B(x, \mathbf{z})$ belongs to $B V_{\text {loc }}\left(\mathbb{R}^{N}\right)$ for all $\mathbf{z} \in \mathbb{R}^{h}$;

(b) $\mathbf{z} \mapsto B(x, \mathbf{z})$ is continuously differentiable in $\mathbb{R}^{h}$ for almost every $x \in \mathbb{R}^{N}$.

We will use the notation $C_{B}$ to denote a Lebesgue negligible set of points such that $B(x, \cdot)$ is $C^{1}$ for all $x \in \mathbb{R}^{N} \backslash C_{B}$.

We assume that $B$ satisfies, besides (a) and (b), the following structural assumptions:

(H1) For some constant $M,\left|\nabla_{\mathbf{z}} B(x, \mathbf{z})\right| \leq M$ for all $x \in \mathbb{R}^{N} \backslash C_{B}$ and $\mathbf{z} \in \mathbb{R}^{h}$.

(H2) For any compact set $H \subset \mathbb{R}^{h}$ there exists a modulus of continuity $\tilde{\omega}_{H}$ independent of $x$ such that

$$
\left|\nabla_{\mathbf{z}} B(x, \mathbf{z})-\nabla_{\mathbf{z}} B\left(x, \mathbf{z}^{\prime}\right)\right| \leq \tilde{\omega}_{H}\left(\left|\mathbf{z}-\mathbf{z}^{\prime}\right|\right)
$$

for all $\mathbf{z}, \mathbf{z}^{\prime} \in H$ and $x \in \mathbb{R}^{N} \backslash C_{B}$.

(H3) For any compact set $H \subset \mathbb{R}^{h}$ there exist a positive Radon measure $\lambda_{H}$ and a modulus of continuity $\omega_{H}$ such that

$$
\left|\widetilde{D}_{x} B(\cdot, \mathbf{z})(A)-\widetilde{D}_{x} B\left(\cdot, \mathbf{z}^{\prime}\right)(A)\right| \leq \omega_{H}\left(\left|\mathbf{z}-\mathbf{z}^{\prime}\right|\right) \lambda_{H}(A)
$$

for all $\mathbf{z}, \mathbf{z}^{\prime} \in H$ and $A \subset \mathbb{R}^{N}$ Borel.

(H4) The measure

$$
\sigma:=\bigvee_{\mathbf{z} \in \mathbb{R}^{h}}\left|D_{x} B(\cdot, \mathbf{z})\right|,
$$

(where $\bigvee$ denotes the least upper bound in the space of nonnegative Borel measures) is finite on compact sets, i.e. it is a Radon measure.

We can now canonically build a countably $\mathcal{H}^{N-1}$-rectifiable set $\mathcal{N}$ containing all jump sets of $B(\cdot, \mathbf{z})$ as follows. Indeed, we define

$$
\mathcal{N}=\left\{x: \limsup _{r \downarrow 0} \frac{\sigma\left(B_{r}(x)\right)}{\omega_{n-1} r^{n-1}}>0\right\} .
$$

It can be checked that $\mathcal{N}$ is $\sigma$-finite with respect to $\mathcal{H}^{N-1}$ and it is countably $\mathcal{H}^{N-1}$ rectifiable (see [1], Section 2, for details).

Theorem 3.2. Let $B$ be satisfying (a), (b), (H1)-(H2)-(H3)-(H4) above. Then for any function $\mathbf{u} \in B V_{\text {loc }}\left(\mathbb{R}^{N} ; \mathbb{R}^{h}\right)$, the function $\mathbf{v}(x):=B(x, \mathbf{u}(x))$ belongs to $B V_{\text {loc }}\left(\mathbb{R}^{N}\right)$ and the following chain rule holds:

(i) (diffuse part) $|D \mathbf{v}| \ll \sigma+|D u|$ and, for any Radon measure $\mu$ such that $\sigma+|D u| \ll$ $\mu$, it holds

$$
\frac{d \widetilde{D} \mathbf{v}}{d \mu}=\frac{d \widetilde{D}_{x} B(\cdot, \tilde{\mathbf{u}}(x))}{d \mu}+\nabla_{\mathbf{z}} \tilde{B}(x, \tilde{\mathbf{u}}(x)) \frac{d \widetilde{D} \mathbf{u}}{d \mu} \quad \mu \text {-a.e. in } \mathbb{R}^{N}
$$


(ii) (jump part) $J_{\mathbf{v}} \subset \mathcal{N} \cup J_{\mathbf{u}}$ and, denoting by $u^{ \pm}(x)$ and $B^{ \pm}(x, \mathbf{z})$ the one-sided traces of $u$ and $B(\cdot, \mathbf{z})$ induced by a suitable orientation of $\mathcal{N} \cup J_{\mathbf{u}}$, it holds

$$
D^{j} \mathbf{v}=\left(B^{+}\left(x, \mathbf{u}^{+}(x)\right)-B^{-}\left(x, \mathbf{u}^{-}(x)\right) \nu_{\mathcal{N} \cup J_{\mathbf{u}}} \mathcal{H}^{N-1}\left\llcorner\left(\mathcal{N} \cup J_{\mathbf{u}}\right)\right.\right.
$$

in the sense of measures.

Moreover for a.e. $x$ the map $y \mapsto B(y, \mathbf{u}(x))$ is approximately differentiable at $x$ and

$$
\nabla \mathbf{v}(x)=\nabla_{x} B(x, \mathbf{u}(x))+\nabla_{\mathbf{z}} B(x, \mathbf{u}(x)) \nabla \mathbf{u}(x) \quad \mathcal{L}^{N} \text {-a.e. in } \mathbb{R}^{N} .
$$

Here the expression

$$
\frac{d \widetilde{D}_{x} B(\cdot, \tilde{\mathbf{u}}(x))}{d \mu}
$$

means the pointwise density of the measure $\widetilde{D}_{x} B(\cdot, \mathbf{z})$ with respect to $\mu$, computed choosing $\mathbf{z}=\tilde{\mathbf{u}}(x)$ (notice that the composition is Borel measurable thanks to the Scorza-Dragoni Theorem and Lemma 3.9 in [1]). Analogously, the expression $\tilde{B}(x, \mathbf{z})$ is well defined at points $x$ such that $x \notin S_{B(\cdot, \mathbf{z})}$ and it can be proved that $\nabla_{\mathbf{z}} \tilde{B}(x, \mathbf{z})$ is well defined for all $\mathbf{z}$ out of a countably $\mathcal{H}^{N-1}$-rectifiable set of points $x$.

\section{A generalization to Divergence-Measure FieldS}

This section is devoted to the proof of the chain rule formula for divergence-measure vector fields (see Theorem 4.7). More precisely, we shall consider the composition $\mathbf{v}(x):=$ $\boldsymbol{B}(x, u(x))$ where $u$ is a function of bounded variation, $\boldsymbol{B}(x, \cdot)$ is of class $C^{1}$ and $\boldsymbol{B}(\cdot, t)$ is a divergence-measure field. (See Section 4.2 for the complete list of assumptions.) In order to obtain the formula in this general setting, we need to assume a-priori the existence of strong traces of $\boldsymbol{B}$ on a countable $\mathcal{H}^{N-1}$-rectifiable universal jump set $\mathcal{N}$. We remark that this assumption is satisfied in the BV setting recalled in Section 3.

Before stating our result, in Section 4.1 we recall some basic facts on divergence-measure fields. Then, after listing all the assumptions in Section 4.2, we prove some preliminary results in Section 4.3. Finally, in Section 4.4 we prove our main uniqueness result.

4.1. Divergence-measure fields. In what follows, since the problem is local we shall assume that $\Omega=\mathbb{R}^{N}$. Moreover, we shall denote by $\mathcal{D} \mathcal{M}^{\infty}$ the space of all vector fields $\boldsymbol{A} \in L_{\mathrm{loc}}^{\infty}\left(\mathbb{R}^{N} ; \mathbb{R}^{N}\right)$ whose divergence in the sense of distribution is a Radon measure with locally finite total variation.

For a vector field $\boldsymbol{A} \in \mathcal{D} \mathcal{M}^{\infty}$ we shall use the usual decomposition of a measure

$$
\operatorname{div} \boldsymbol{A}=: \operatorname{div}^{a} \boldsymbol{A} \mathcal{L}^{N}+\operatorname{div}^{s} \boldsymbol{A},
$$

where $\operatorname{div}^{a} \boldsymbol{A} \mathcal{L}^{N}$ is the absolutely continuous part and $\operatorname{div}^{s} \boldsymbol{A}$ is the singular part of $\operatorname{div} \boldsymbol{A}$ with respect to the Lebesgue measure. On the other hand, Chen and Frid in [7] proved that if $\boldsymbol{A} \in \mathcal{D} \mathcal{M}^{\infty}$ then $\operatorname{div} \boldsymbol{A} \ll \mathcal{H}^{N-1}$, hence the singular part can be further decomposed as

$$
\operatorname{div}^{s} \boldsymbol{A}=: \operatorname{div}^{c} \boldsymbol{A}+\operatorname{div}^{j} \boldsymbol{A},
$$

where $\operatorname{div}^{c} \boldsymbol{A}(C)=0$ if $C$ has $\sigma$-finite $\mathcal{H}^{N-1}$-measure. We shall also use the diffuse part $\widetilde{\operatorname{div}} \boldsymbol{A}:=\operatorname{div}^{a} \boldsymbol{A} \mathcal{L}^{N}+\operatorname{div}^{c} \boldsymbol{A}$ of the measure $\operatorname{div} \boldsymbol{A}$.

In the following, we shall denote by $\mathcal{S D} \mathcal{M}^{\infty}$ the space of all vector fields $\boldsymbol{A} \in \mathcal{D} \mathcal{M}^{\infty}$ such that $\operatorname{div}^{c} \boldsymbol{A}=0$. 
Given a domain $\Omega \subset \mathbb{R}^{N}$ of class $C^{1}$, we can define the trace of the normal component of $\boldsymbol{A}$ on $\partial \Omega$ as a distribution as follows:

$$
\langle\operatorname{Tr}(\boldsymbol{A}, \partial \Omega), \varphi\rangle:=\int_{\Omega} \nabla \varphi \cdot \boldsymbol{A} d x+\int_{\Omega} \varphi d \operatorname{div} \boldsymbol{A}, \quad \forall \varphi \in C_{c}^{\infty}\left(\mathbb{R}^{N}\right)
$$

$($ see $[6,7])$.

This notion of distributional trace can be extended to any $\mathcal{H}^{N-1}$-rectifiable set $\mathcal{J} \subset \mathbb{R}^{N}$. In particular, it is possible to define the traces $\operatorname{Tr}^{ \pm}(\boldsymbol{A}, \mathcal{J})$ in such a way that $\operatorname{Tr}^{-}(\boldsymbol{A}, \partial \Omega)=$ $\operatorname{Tr}(\boldsymbol{A}, \partial \Omega)$ for every $\Omega \Subset \mathbb{R}^{N}$ of class $C^{1}$ (see [2], Definition 3.3).

Unfortunately, these distributional traces are in general too weak to be used in a chain rule (see the discussion in [2] and [4]).

For our purposes we need the notion of (strong) traces given below.

Definition 4.1 (Traces). Let $u \in L_{\text {loc }}^{\infty}\left(\mathbb{R}^{N}\right)$ and let $\mathcal{J} \subset \mathbb{R}^{N}$ be a countably $\mathcal{H}^{N-1}$ rectifiable set oriented by a normal vector field $\nu$. We say that two Borel functions $u^{ \pm}: \mathcal{J} \rightarrow \mathbb{R}$ are the traces of $u$ on $\mathcal{J}$ if for $\mathcal{H}^{N-1}$-almost every $x \in \mathcal{J}$ it holds

$$
\lim _{r \rightarrow 0^{+}} \int_{B_{r}^{ \pm}(x)}\left|u(y)-u^{ \pm}(x)\right| d y=0,
$$

where $B_{r}^{ \pm}(x):=B_{r}(x) \cap\left\{y \in \mathbb{R}^{N}: \pm\langle y-x, \nu(x)\rangle \geq 0\right\}$.

The following result is a particular case of Lemma 3.1 in [11].

Lemma 4.2. Let $\mathcal{J}$ be a countably $\mathcal{H}^{N-1}$-rectifiable set with oriented normal vector $\nu$. Let $\boldsymbol{A} \in \mathcal{D} \mathcal{M}^{\infty}$ and assume that $\boldsymbol{A}$ admits traces $\boldsymbol{A}^{ \pm}$on $\mathcal{J}$ for $\mathcal{H}^{N-1}$ almost every $z \in \mathcal{J}$. Then it holds

$$
\operatorname{div} \boldsymbol{A}\left\llcorner\mathcal{J}=\left\langle\boldsymbol{A}^{+}-\boldsymbol{A}^{-}, \nu\right\rangle \mathcal{H}^{N-1}\llcorner\mathcal{J}\right.
$$

4.2. Assumptions on the vector field $\boldsymbol{b}$. Let $\boldsymbol{b}: \mathbb{R}^{N} \times \mathbb{R} \rightarrow \mathbb{R}^{N}$ be a locally bounded Borel function satisfying the following conditions:

(i) for $\mathcal{L}^{N}$-a.e. $x \in \mathbb{R}^{N}$ the function $\boldsymbol{b}(x, \cdot)$ is continuous in $\mathbb{R}$, uniformly w.r.t. $x$;

(ii) for $\mathcal{L}^{1}$-a.e. $t \in \mathbb{R}$ the function $\boldsymbol{b}(\cdot, t) \in \mathcal{D} \mathcal{M}^{\infty}$;

(iii) the measure

$$
\sigma:=\bigvee_{t \in \mathbb{R}}\left|\operatorname{div}_{x} \boldsymbol{b}(\cdot, t)\right|
$$

is a Radon measure;

(iv) for any compact set $H \subset \mathbb{R}$ there exist a positive Radon measure $\lambda_{H}$ and a modulus of continuity $\omega_{H}$ such that

$$
\left|\widetilde{\operatorname{div}_{x}} \boldsymbol{b}(\cdot, t)(C)-\widetilde{\operatorname{div}_{x}} \boldsymbol{b}(\cdot, w)(C)\right| \leq \omega_{H}(|t-w|) \lambda_{H}(C)
$$

for all $t, w \in H$ and $C \subset \mathbb{R}^{N}$ Borel.

Let us define the singular set

$$
\mathcal{N}=\left\{x \in \mathbb{R}^{N}: \liminf _{r \rightarrow 0} \frac{\sigma\left(B_{r}(x)\right)}{r^{N-1}}>0\right\} .
$$

We assume that

(v) $\mathcal{N}$ is a countably $\mathcal{H}^{N-1}$-rectifiable set and $\mathcal{H}^{N-1}(\mathcal{N} \cap K)<+\infty$ for every compact set $K \subseteq \mathbb{R}^{N}$. 
In the following, $\nu$ will always denote an oriented normal vector field on $\mathcal{N}$. We remark that, in the setting of Theorem 3.2, the rectifiability of the singular set $\mathcal{N}$ follows from (i)-(iv).

Furthermore we assume that

(vi) for every $t \in \mathbb{R}$ and for $\mathcal{H}^{N-1}$ almost every $x \in \mathbb{R}^{N} \backslash \mathcal{N}$ there exists the limit

$$
\widetilde{\boldsymbol{b}}(x, t)=\lim _{r \rightarrow 0} f_{B_{r}(x)} \boldsymbol{b}(y, t) d y .
$$

Without loss of generality we shall always assume that $\boldsymbol{b}(x, t)=\widetilde{\boldsymbol{b}}(x, t)$ on points $(x, t)$ where (15) holds.

By using (vi), as in $\left[1\right.$, Section 3], we can prove that there exists a set $\mathcal{N}_{0}$ with $\mathcal{H}^{N-1}\left(\mathcal{N}_{0}\right)=0$ such that for every point $x \in \mathbb{R}^{N} \backslash\left(\mathcal{N} \cup \mathcal{N}_{0}\right)$ and every $t \in \mathbb{R}$ there exists the limit

$$
\widetilde{\boldsymbol{b}}(x, t)=\lim _{r \rightarrow 0} f_{B_{r}(x)} \boldsymbol{b}(y, t) d y .
$$

Moreover we consider the following assumption on the traces of the vector field $\boldsymbol{b}$.

(vii) For every $t \in \mathbb{R}$, the function $\boldsymbol{b}(\cdot, t)$ admits (strong) traces $\boldsymbol{b}^{ \pm}(\cdot, t)$ on $\mathcal{N}$.

In what follows, we shall use the notation $\beta^{ \pm}(x, t):=\left\langle\boldsymbol{b}^{ \pm}(x, t), \nu(x)\right\rangle, t \in \mathbb{R}, x \in \mathcal{N}$.

Remark 4.3. By assumption (ii) it follows that, for every $t \in \mathbb{R}$, the vector field $\boldsymbol{b}(\cdot, t)$ admits distributional traces $\operatorname{Tr}^{ \pm}(\boldsymbol{b}(\cdot, t), \mathcal{N})$ on $\mathcal{N}$ in the sense of Anzellotti (see [6, 7]). Nevertheless, this notion of trace is too weak in order to obtain the chain rule formula.

As in [1, Prop. 3.2(ii)], it can be proved that there exists a Borel set $\mathcal{N}_{1} \subseteq \mathcal{N}$ such that, for every $x \in \mathcal{N}_{1}$, the traces $\boldsymbol{b}^{ \pm}(x, t)$ are defined for every $t \in \mathbb{R}$ and are continuous in $t$.

By (vii) and Proposition 4.2 we have

$$
\frac{d \operatorname{div}_{x}^{j} \boldsymbol{b}(\cdot, t)}{d \mathcal{H}^{N-1}}(x)=\beta^{+}(x, t)-\beta^{-}(x, t)
$$

for every $t \in \mathbb{R}$ and for $\mathcal{H}^{N-1}$ a.e. $x \in \mathcal{N}$.

If assumptions (i)-(vii) hold, then for every $t \in \mathbb{R}$ the decomposition formula

$$
\left(\operatorname{div}_{x} \boldsymbol{b}\right)(\cdot, t)=\left(\operatorname{div}_{x}^{a} \boldsymbol{b}\right)(x, t) \mathcal{L}^{N}+\frac{\operatorname{div}_{x}^{c} \boldsymbol{b}(\cdot, t)}{d \sigma}(x) \sigma+\left[\beta^{+}(x, t)-\beta^{-}(x, t)\right] \mathcal{H}^{N-1}\llcorner\mathcal{N}
$$

holds in the sense of measures and there exists a Borel set $\mathcal{N}_{2} \subseteq \mathbb{R}^{N}$ with $\sigma\left(\mathcal{N}_{2}\right)=0$ such that the following limit

$$
\lim _{r \downarrow 0} \frac{\widetilde{\operatorname{div}}_{x} \boldsymbol{b}(\cdot, t)\left(B_{r}(x)\right)}{\sigma\left(B_{r}(x)\right)}=\frac{d \widetilde{\operatorname{div}}_{x} \boldsymbol{b}(\cdot, t)}{d \sigma}(x)
$$

exists for every $x \in \mathbb{R}^{N} \backslash \mathcal{N}_{2}$ and for every $t \in \mathbb{R}$ and this equality holds, where $\frac{d \operatorname{div}_{x} \boldsymbol{b}(\cdot, t)}{d \sigma}(x)$ is the Radon-Nikodým derivative at $x$ of the measure $\operatorname{div}_{x} \boldsymbol{b}(\cdot, t)$ w.r.t. $\sigma$. In particular we have that there exists a Borel set $\mathcal{N}_{3} \subseteq \mathbb{R}^{N}$ with $\mathcal{L}^{N}\left(\mathcal{N}_{3}\right)=0$ such that the following limit

$$
\lim _{r \downarrow 0} \frac{\operatorname{div}_{x}^{a} \boldsymbol{b}(\cdot, t)\left(B_{r}(x)\right)}{\mathcal{L}^{N}\left(B_{r}(x)\right)}=\frac{d \operatorname{div}_{x}^{a} \boldsymbol{b}(\cdot, t)}{d \mathcal{L}^{N}}(x)
$$

exists for every $x \in \mathbb{R}^{N} \backslash \mathcal{N}_{3}$ and for every $t \in \mathbb{R}$ and this equality holds, where $\frac{d \operatorname{div}_{x}^{a} \boldsymbol{b}(\cdot, t)}{d \sigma}(x)$ is the Radon-Nikodým derivative at $x$ of the measure $\operatorname{div}_{x}^{a} \boldsymbol{b}(\cdot, t)$ w.r.t. $\mathcal{L}^{N}$. Similarly, there 
exists a Borel set $\mathcal{N}_{4} \subseteq \mathbb{R}^{N}$ with $\sigma\left(\mathcal{N}_{4}\right)=0$ such that the following limit

$$
\lim _{r \downarrow 0} \frac{\operatorname{div}_{x}^{c} \boldsymbol{b}(\cdot, t)\left(B_{r}(x)\right)}{\sigma\left(B_{r}(x)\right)}=\frac{d \operatorname{div}_{x}^{c} \boldsymbol{b}(\cdot, t)}{d \sigma}(x)
$$

exists for every $x \in \mathbb{R}^{N} \backslash \mathcal{N}_{4}$ and for every $t \in \mathbb{R}$ and this equality holds, where $\frac{d \operatorname{div}_{x}^{c} \boldsymbol{b}(\cdot, t)}{d \sigma}(x)$ is the Radon-Nikodým derivative at $x$ of the measure $\operatorname{div}_{x}^{c} \boldsymbol{b}(\cdot, t)$ w.r.t. $\sigma$.

4.3. Preliminary results. Let us define

$$
\boldsymbol{B}(x, t)=\int_{0}^{t} \boldsymbol{b}(x, w) d w .
$$

Proposition 4.4. Let $\boldsymbol{b}: \mathbb{R}^{N} \times \mathbb{R} \rightarrow \mathbb{R}^{N}$ be a Borel function satisfying (i)-(vii). We have that

(a) for every $t \in \mathbb{R}$ the function $x \mapsto \boldsymbol{B}(x, t) d t$ belongs to $\mathcal{D} \mathcal{M}^{\infty}$;

(b) for every $t \in \mathbb{R}$

$$
\operatorname{div}_{x} \boldsymbol{B}(x, t) \ll \sigma
$$

(c) the equality

$$
\operatorname{div}_{x} \boldsymbol{B}(x, t)=\left[\int_{0}^{t} \frac{d \operatorname{div}_{x} \boldsymbol{b}}{d \sigma}(x, w) d w\right] d \sigma
$$

holds in the sense of measures for every $t \in \mathbb{R}$.

Proof. Since

$$
\left|\frac{d \operatorname{div}_{x} \boldsymbol{b}}{d \sigma}(x, w)\right| \leq 1,
$$

for every test function $\phi \in C_{0}^{1}\left(\mathbb{R}^{N}\right)$, for $\mathcal{H}^{N-1}$ a.e. $x \in \mathbb{R}^{N}$ and for every $t \in \mathbb{R}$, we have

$$
\begin{aligned}
& \int_{\mathbb{R}^{N}} \nabla \phi(x) \boldsymbol{B}(x, t) d x=\int_{0}^{t}\left[\int_{\mathbb{R}^{N}} \nabla \phi(x) \boldsymbol{b}(x, w) d x\right] d w \\
= & -\int_{0}^{t}\left[\int_{\mathbb{R}^{N}} \phi(x) d \operatorname{div}_{x} \boldsymbol{b}(\cdot, w)\right] d w=-\int_{0}^{t}\left[\int_{\mathbb{R}^{N}} \phi(x) \frac{d \operatorname{div}_{x} \boldsymbol{b}}{d \sigma}(x, w) d \sigma\right] d w \\
= & -\int_{\mathbb{R}^{N}} \phi(x)\left[\int_{0}^{t} \frac{d \operatorname{div}_{x} \boldsymbol{b}}{d \sigma}(x, w) d w\right] d \sigma .
\end{aligned}
$$

Corollary 4.5. Under the previous assumptions, we have

$$
\operatorname{div}_{x}^{a} \boldsymbol{B}(x, t)=\int_{0}^{t} \frac{d \operatorname{div}_{x}^{a} \boldsymbol{b}}{d \mathcal{L}^{N}}(x, w) d w \quad \forall x \in \mathbb{R}^{N} \backslash \mathcal{N}_{3},
$$

and

in the sense of measures.

$$
\operatorname{div}_{x}^{c} \boldsymbol{B}(x, t)=\left[\int_{0}^{t} \frac{d \operatorname{div}_{x}^{c} \boldsymbol{b}}{d \sigma}(x, w) d w\right] d \sigma
$$

Corollary 4.6. Let $\boldsymbol{b}$ be a Borel function satisfying (i)-(vii) and let $\boldsymbol{B}$ be the vector field defined in (18). Then it holds:

(a) For every $x \in \mathcal{N} \backslash \mathcal{N}_{1}$ and for every $w \in \mathbb{R}$ one has

$$
\lim _{r \downarrow 0} f_{B_{r}^{ \pm}(x)}\left|\boldsymbol{B}(y, w)-\boldsymbol{B}^{ \pm}(x, w)\right| d y=0,
$$


where

$$
\boldsymbol{B}^{ \pm}(x, w):=\int_{0}^{w} \boldsymbol{b}^{ \pm}(x, t) d t .
$$

(b) For every $x \in \mathbb{R}^{N} \backslash\left(\mathcal{N} \cup \mathcal{N}_{1}\right)$ and every $w \in \mathbb{R}$ one has

$$
\lim _{r \downarrow 0} f_{B_{r}(x)}|\boldsymbol{B}(y, w)-\widetilde{B}(x, w)| d y=0,
$$

where

$$
\widetilde{\boldsymbol{B}}(x, w):=\int_{0}^{w} \boldsymbol{b}(x, t) d t .
$$

(c) The equality

$$
\begin{aligned}
\operatorname{div}_{x} \boldsymbol{B}(x, t)= & {\left[\int_{0}^{w} \nabla_{x} \boldsymbol{b}(x, t) d t\right] d \mathcal{L}^{N}+\left[\int_{0}^{w} \frac{d D_{x}^{c} \boldsymbol{b}}{d \sigma}(x, t) d t\right] d \sigma } \\
& +\left\langle\boldsymbol{B}^{+}(x, t)-\boldsymbol{B}^{-}(x, t), \nu(x)\right\rangle d \mathcal{H}^{N-1} \mathbf{L} \mathcal{N}
\end{aligned}
$$

holds in the sense of measures.

Proof. (a) By (vii) for every $x \in \mathcal{N} \backslash \mathcal{N}_{1}$ and for every $w \in \mathbb{R}$ we have

$$
\begin{aligned}
& \lim _{r \downarrow 0} f_{B_{r}^{ \pm}(x)}\left|\int_{0}^{w} \boldsymbol{b}(y, t) d t-\int_{0}^{w} \boldsymbol{b}^{ \pm}(x, t) d t\right| d y \\
& \leq \int_{0}^{w} \lim _{r \downarrow 0} f_{B_{r}^{ \pm}(x)}\left|\boldsymbol{b}(y, t)-\boldsymbol{b}^{ \pm}(x, t)\right| d y d t=0 .
\end{aligned}
$$

(b) Similarly for every $x \in \mathbb{R}^{N} \backslash\left(\mathcal{N} \cup \mathcal{N}_{1}\right)$ and for every $w \in \mathbb{R}$ we have

$$
\begin{aligned}
& \lim _{r \downarrow 0} f_{B_{r}(x)}\left|\int_{0}^{w} \boldsymbol{b}(y, t) d t-\int_{0}^{w} \widetilde{\boldsymbol{b}}(x, t) d t\right| d y \\
& \leq \int_{0}^{w} \lim _{r \downarrow 0} f_{B_{r}(x)}|\boldsymbol{b}(y, t)-\widetilde{\boldsymbol{b}}(x, t)| d y d t=0 .
\end{aligned}
$$

(c) Follows from (a), (b) and Corollary 4.5.

\subsection{Main result.}

Theorem $4.7\left(\mathcal{D} \mathcal{M}^{\infty}\right.$-dependence). Let $\boldsymbol{b}: \mathbb{R}^{N} \times \mathbb{R} \rightarrow \mathbb{R}^{N}$ be a locally bounded Borel function satisfying conditions (i)-(vii). Then, for every $u \in B V_{\mathrm{loc}}\left(\mathbb{R}^{N}\right) \cap L_{\mathrm{loc}}^{\infty}\left(\mathbb{R}^{N}\right)$, the function $\mathbf{v}: \mathbb{R}^{N} \rightarrow \mathbb{R}^{N}$, defined by

$$
\mathbf{v}(x):=\boldsymbol{B}(x, u(x)),
$$

belongs to $\mathcal{D} \mathcal{M}^{\infty}$ and for any $\phi \in C_{0}^{1}\left(\mathbb{R}^{N}\right)$ we have

$$
\begin{aligned}
& \int_{\mathbb{R}^{N}}\langle\nabla \phi(x), \mathbf{v}(x)\rangle d x= \\
& \quad-\int_{\mathbb{R}^{N}} \phi(x) \operatorname{div}_{x}^{a} \boldsymbol{B}(x, u(x)) d x-\int_{\mathbb{R}^{N}} \phi(x) \frac{\operatorname{div}_{x}^{c} \boldsymbol{B}}{d \sigma}(x, \widetilde{u}(x)) d \sigma \\
& -\int_{\mathbb{R}^{N}} \phi(x)\langle\boldsymbol{b}(x, \widetilde{u}(x)), \nabla u(x)\rangle d x-\int_{\mathbb{R}^{N}} \phi(x)\left\langle\boldsymbol{b}(x, \widetilde{u}(x)), \frac{D^{c} u}{|D u|}(x)\right\rangle d|D u|(x) \\
& -\int_{\mathcal{N} \cup J_{u}} \phi(x)\left\langle\boldsymbol{B}^{+}\left(x, u^{+}(x)\right)-\boldsymbol{B}^{-}\left(x, u^{-}(x)\right), \nu(x)\right\rangle d \mathcal{H}^{N-1} .
\end{aligned}
$$


Proof. Step 1. Let us fix a test function $\phi \in C_{0}^{1}\left(\mathbb{R}^{N}\right)$. We claim that

$$
\begin{aligned}
\int_{\mathbb{R}^{N}}\langle\nabla \phi(x), \mathbf{v}(x)\rangle d x= & -\int_{\mathbb{R}} d t \int_{\mathbb{R}^{N}} \operatorname{sign}(t) \chi_{\Omega_{u, t}}^{*} \phi(x) d \operatorname{div}_{x} \boldsymbol{b}(x, t) \\
& -\int_{\mathbb{R}^{N}} \phi(x)\langle\boldsymbol{b}(x, \widetilde{u}(x)), \nabla u(x)\rangle d x \\
& -\int_{\mathbb{R}^{N}} \phi(x)\left\langle\boldsymbol{b}(x, \widetilde{u}(x)), \frac{D^{c} u}{|D u|}(x)\right\rangle d|D u|(x) \\
& -\frac{1}{2} \int_{\mathcal{N} \cup J_{u}} \phi(x)\left[\int_{u^{-}(x)}^{u^{+}(x)}\left[\beta^{+}(x, t)+\beta^{-}(x, t)\right] d t\right] d \mathcal{H}^{N-1},
\end{aligned}
$$

where $\Omega_{u, t}=\left\{x \in \mathbb{R}^{N}: t\right.$ belongs to the segment of endpoints 0 and $\left.u(x)\right\}$ and $\chi_{\Omega_{u, t}}^{*}$ is the precise representative of the $B V$ function $\chi_{\Omega_{u, t}}$.

In order to prove the claim, it is enough to use a regularization argument as in [15]. More precisely, if $\boldsymbol{b}_{\epsilon}(\cdot, t):=\rho_{\epsilon} * \boldsymbol{b}(\cdot, t)$ denotes the standard regularization of $\boldsymbol{b}(\cdot, t)$, and $\mathbf{v}_{\epsilon}(x):=\int_{0}^{u(x)} \boldsymbol{b}_{\epsilon}(x, t) d t$, then

$$
\begin{aligned}
\int_{\mathbb{R}^{N}}\left\langle\nabla \phi(x), \mathbf{v}_{\epsilon}(x)\right\rangle d x= & -\int_{\mathbb{R}^{N}} \phi(x) \int_{0}^{u(x)} \operatorname{div}_{x} \boldsymbol{b}_{\epsilon}(x, t) d t \\
& -\int_{\mathbb{R}^{N}} \phi(x)\left\langle\boldsymbol{b}_{\epsilon}(x, u), \nabla u\right\rangle d x \\
& -\int_{\mathbb{R}^{N}} \phi(x)\left\langle\boldsymbol{b}_{\epsilon}(x, \widetilde{u}(x)), \frac{D^{c} u}{|D u|}(x)\right\rangle d|D u|(x) \\
& -\int_{\mathcal{N} \cup J_{u}} \phi(x) \int_{u^{-}(x)}^{u^{+}(x)}\left\langle\boldsymbol{b}_{\epsilon}(x, t), \nu(x)\right\rangle d t d \mathcal{H}^{N-1}(x),
\end{aligned}
$$

and the claim follows by passing to the limit as $\epsilon \rightarrow 0^{+}$, observing that, by (vii), for every $t \in \mathbb{R}$ it holds

$$
\lim _{\epsilon \rightarrow 0^{+}}\left\langle\boldsymbol{b}_{\epsilon}(x, t), \nu(x)\right\rangle=\frac{\beta^{+}(x, t)+\beta^{-}(x, t)}{2} \quad \text { for } \mathcal{H}^{N-1}-\text { a.e. } x \in \mathcal{N} \text {. }
$$

(For details see the proofs of Theorems 1.1 and 3.4 in [15].)

Step 2. We assume for simplicity that $u \geq 0$. We claim that

$$
\begin{aligned}
& \int_{\mathbb{R}}\left[\int_{\mathbb{R}^{N}} \operatorname{sgn}(t) \phi(x) \chi_{\Omega_{u, t}}^{*}(x) d \operatorname{div}_{x} \boldsymbol{b}((x, t)] d t\right. \\
& =\int_{\mathbb{R}^{N}} \phi(x) \operatorname{div}_{x}^{a} \boldsymbol{B}(x, u(x)) d x-\int_{\mathbb{R}^{N}} \phi(x) \frac{d \operatorname{div}_{x}^{c} \boldsymbol{B}}{d \sigma}(x, \widetilde{u}(x)) d \sigma \\
& \quad+\frac{1}{2} \int_{\mathcal{N} \cup J_{u}} \phi(x)\left[\int_{0}^{u^{+}(x)}\left[\beta^{+}(x, t)-\beta^{-}(x, t)\right] d t+\int_{0}^{u^{-}(x)}\left[\beta^{+}(x, t)-\beta^{-}(x, t)\right] d t\right] d \mathcal{H}^{N-1} .
\end{aligned}
$$


By using Lemma 2.2 in [15] and by the decomposition formula (17), we have

$$
\begin{aligned}
& \int_{-\infty}^{+\infty}\left[\int_{\mathbb{R}^{N}} \operatorname{sgn}(t) \phi(x) \chi_{\Omega_{u, t}}^{*} d \operatorname{div}_{x} \boldsymbol{b}(x, t)\right] d t=\int_{0}^{+\infty}\left[\int_{\mathbb{R}^{N}} \phi(x) \chi_{\{u>t\}}^{*} d \operatorname{div}_{x} \boldsymbol{b}(x, t)\right] d t \\
& =\int_{0}^{+\infty}\left[\int_{\mathbb{R}^{N}} \phi(x) \chi_{\{u>t\}} \operatorname{div}_{x}^{a} \boldsymbol{b}(x, t) d x\right] d t+\int_{0}^{+\infty}\left[\int_{\mathbb{R}^{N}} \phi(x) \chi_{\{\tilde{u}>t\}} \frac{d \operatorname{div}_{x}^{c} \boldsymbol{b}}{d \sigma}(x, t) d \sigma\right] d t \\
& +\int_{0}^{+\infty}\left[\int_{\mathcal{N} \cup J_{u}} \phi(x) \frac{1}{2}\left[\chi_{\left\{u^{+}>t\right\}}+\chi_{\left\{u^{-}>t\right\}}\right]\left[\beta^{+}(x, t)-\beta^{-}(x, t)\right] d \mathcal{H}^{N-1}\right] d t .
\end{aligned}
$$

By using Corollary 4.5, the claim is proved.

Finally, the thesis follows from Step 1 and Step 2, observing that

$$
\begin{aligned}
& \frac{1}{2} \int_{u^{-}(x)}^{u^{+}(x)}\left[\beta^{+}(x, t)+\beta^{-}(x, t)\right] d t \\
& +\frac{1}{2}\left[\int_{0}^{u^{+}(x)}\left[\beta^{+}(x, t)-\beta^{-}(x, t)\right] d t+\int_{0}^{u^{-}(x)}\left[\beta^{+}(x, t)-\beta^{-}(x, t)\right] d t\right] \\
& =\int_{0}^{u^{+}(x)} \beta^{+}(x, t) d t-\int_{0}^{u^{-}(x)} \beta^{-}(x, t) d t .
\end{aligned}
$$

Corollary 4.8. Let $h \in C^{1}(\mathbb{R})$ be a function with bounded derivative, let $\mathbf{A} \in \mathcal{D} \mathcal{M}^{\infty}$ and let

$$
\mathcal{N}=\left\{x \in \mathbb{R}^{N}: \liminf _{r \rightarrow 0} \frac{|\operatorname{div} \mathbf{A}|\left(B_{r}(x)\right)}{r^{N-1}}>0\right\} .
$$

Assume that:

(a) $\mathcal{N}$ is a countably $\mathcal{H}^{N-1}$-rectifiable set and $\mathcal{H}^{N-1}(\mathcal{N} \cap K)<+\infty$ for every compact set $K \subseteq \mathbb{R}^{N}$.

(b) For every $x \in \mathbb{R}^{N} \backslash \mathcal{N}$, one has $\mathbf{A}(x)=\widetilde{\mathbf{A}}(x)$.

(c) The vector field $\mathbf{A}$ admits strong traces $\mathbf{A}^{ \pm}$on $\mathcal{N}$.

Then, for every $u \in B V\left(\mathbb{R}^{N}\right) \cap L^{\infty}\left(\mathbb{R}^{N}\right)$, the function $\mathbf{v}: \mathbb{R}^{N} \rightarrow \mathbb{R}^{N}$, defined by

$$
\mathbf{v}(x):=\mathbf{A}(x) h(u(x)),
$$

belongs to $\mathcal{D} \mathcal{M}^{\infty}$ and for any $\phi \in C_{0}^{1}\left(\mathbb{R}^{N}\right)$ we have

$$
\begin{aligned}
& \int_{\mathbb{R}^{N}}\langle\nabla \phi(x), \mathbf{v}(x)\rangle d x= \\
- & \int_{\mathbb{R}^{N}} \phi(x) \operatorname{div}^{a} \mathbf{A}(x) h(u(x)) d x-\int_{\mathbb{R}^{N}} \phi(x) \frac{\operatorname{div}^{c} \mathbf{A}}{d \sigma}(x) h(\widetilde{u}(x)) d \sigma \\
- & \int_{\mathbb{R}^{N}} \phi(x) h^{\prime}(\widetilde{u}(x))\langle\mathbf{A}(x), \nabla u(x)\rangle d x-\int_{\mathbb{R}^{N}} \phi(x) h^{\prime}(\widetilde{u}(x))\left\langle\mathbf{A}(x), \frac{D^{c} u}{|D u|}(x)\right\rangle d|D u|(x) \\
- & \int_{\mathcal{N} \cup J_{u}} \phi(x)\left[h\left(u^{+}(x)\right)\left\langle\mathbf{A}^{+}(x), \nu(x)\right\rangle-h\left(u^{-}(x)\right)\left\langle\mathbf{A}^{-}(x), \nu(x)\right\rangle\right] d \mathcal{H}^{N-1} .
\end{aligned}
$$

Remark 4.9. The theory of divergence-measure vector fields is due to G. Anzellotti [6] (see also G.-Q. Chen and H. Frid [7] for its generalization and [20]). He introduced the "dot product" of a bounded vector field $\boldsymbol{A}$, whose divergence is a Radon measure, and the gradient $D u$ of $u \in B V(\Omega)$ through a pairing $(\boldsymbol{A}, D u)$ which defines a Radon measure. 
He also defined the normal trace of a vector field through the boundary and establish a generalized Gauss-Green formula.

Consider now $\mu=\operatorname{div} \boldsymbol{A}$ with $\boldsymbol{A} \in \mathcal{D} \mathcal{M}^{\infty}$ and let $u \in B V_{\mathrm{loc}}\left(\mathbb{R}^{N}\right) \cap L_{\mathrm{loc}}^{\infty}\left(\mathbb{R}^{N}\right)$. The distribution defined by the following expression

$$
\langle(\boldsymbol{A}, D u), \varphi\rangle=-\int u^{*} \varphi d \mu-\int u \boldsymbol{A} \cdot \nabla \varphi, \quad \varphi \in C_{0}^{\infty}\left(\mathbb{R}^{N}\right),
$$

is actually a Radon measure and its total variation $|(\boldsymbol{A}, D u)|$ is absolutely continuous with respect to the measure $|D u|$. Therefore the following Anzellotti formula holds

$$
\operatorname{div}(u \boldsymbol{A})=u^{*} \operatorname{div} \boldsymbol{A}+(\boldsymbol{A}, D u) .
$$

in the sense of measures. Using the distributional normal trace defined in (13), the following Green formula holds

$$
\int_{\Omega} u^{*} d \mu+\int_{\Omega}(\boldsymbol{A}, D u)=\int_{\partial \Omega} \operatorname{Tr}(\boldsymbol{A}, \partial \Omega) u d \mathcal{H}^{N-1} .
$$

We remark that, if we apply (19) to a vector field $\boldsymbol{A} \in \mathcal{D} \mathcal{M}^{\infty}(\Omega)$ and the constant $u \equiv 1$, since $(\boldsymbol{A}, D u)=0$ we obtain

$$
\int_{\Omega} \operatorname{div} \boldsymbol{A}=\int_{\partial \Omega} \operatorname{Tr}(\boldsymbol{A}, \partial \Omega) d \mathcal{H}^{N-1} .
$$

Remark 4.10. The case $u \in W^{1,1}$ in Theorem 4.7 has been already treated in [15] (see also [16]). The representation formula in Theorem 4.7 can be written as the following equality in the sense of measures

$$
\begin{aligned}
\operatorname{div}(\boldsymbol{B}(x, u(x))) & =\left.\operatorname{div}_{x} \boldsymbol{B}(x, t)\right|_{t=u(x)} \\
& +\langle\boldsymbol{b}(x, \widetilde{u}(x)), \widetilde{D} u\rangle+\left\langle\boldsymbol{B}^{*}\left(x, u^{+}\right)-\boldsymbol{B}^{*}\left(x, u^{-}\right), \nu\right\rangle \mathcal{H}^{N-1}\left\llcorner\mathcal{N} \cup J_{u}\right.
\end{aligned}
$$

with the compact notation

$$
\left.\operatorname{div}_{x} \boldsymbol{B}(\cdot, t)\right|_{t=u(x)}=\frac{1}{2}\left[\operatorname{div}_{x} \boldsymbol{B}\left(\cdot, u^{+}(x)\right)+\operatorname{div}_{x} \boldsymbol{B}\left(\cdot, u^{-}(x)\right)\right] .
$$

Remark 4.11. The formula in Corollary 4.8 can be written as the following equality in the sense of measures:

$$
\operatorname{div}(\mathbf{A}(x) h(u(x)))=\operatorname{div} \mathbf{A}(x) h(u)^{*}(x)+h^{\prime}(u(x))\langle\mathbf{A}(x), D u\rangle .
$$

If $u \in B V$ and $u \mathbf{A} \in \mathcal{D} \mathcal{M}^{\infty}$, then

$$
\langle\mathbf{A}(x), D u\rangle=-u^{*} \operatorname{div} \mathbf{A}+\operatorname{div}(u \mathbf{A}) .
$$

Hence the following formula holds

$$
\begin{aligned}
\operatorname{div}(\mathbf{A}(x) h(u)) & =\operatorname{div} \mathbf{A} h(u)^{*}+h^{\prime}(u)[-u \operatorname{div} \mathbf{A}+\operatorname{div}(u \mathbf{A})] \\
& =\left[h(u)^{*}-u h^{\prime}(u)\right] \operatorname{div} \mathbf{A}+h^{\prime}(u) \operatorname{div}(u \mathbf{A}) .
\end{aligned}
$$

(For a similar formula when $u$ is not a function of bounded variation see [4].)

If $\boldsymbol{B}(x, u(x))=\mathbf{A}(x) u(x)$, then we obtain the Anzellotti formula (18). 


\section{Applications to Conservation laws}

Let us consider the multidimensional scalar conservation law with discontinuous flux

$$
u_{t}+\operatorname{div} \boldsymbol{B}(x, u)=0, \quad(t, x) \in(0,+\infty) \times \mathbb{R}^{N},
$$

where, as in the previous section, the vector field $\boldsymbol{B}$ is defined as in (18) by $\boldsymbol{B}(x, t):=$ $\int_{0}^{t} \boldsymbol{b}(x, w) d w$. Here the Borel function $\boldsymbol{b}: \mathbb{R}^{N} \times \mathbb{R} \rightarrow \mathbb{R}^{N}$ satisfies slightly stronger assumptions compared with (i)-(vii) of Section 4.

We are interested in proving the Kato contraction property for entropy solutions, in the sense of Definition 5.4, of (22), see Theorem 5.5. We stress here that, as in [10], our definition of entropy solution is restricted to BV functions.

For the sake of completeness we collect the assumptions on $\boldsymbol{b}$ here. (A prime denotes the fact that the assumption has been modified with respect to the corresponding one listed in Section 4.2.)

(i) for $\mathcal{L}^{N}$-a.e. $x \in \mathbb{R}^{N}$ the function $\boldsymbol{b}(x, \cdot)$ is continuous in $\mathbb{R}$ uniformly w.r.t. $x$;

(ii') for $\mathcal{L}^{1}$-a.e. $t \in \mathbb{R}$ the function $\boldsymbol{b}(\cdot, t)$ belongs to $\mathcal{S D} \mathcal{M}^{\infty}$;

(iii) the measure $\sigma$ defined in (14) is a Radon measure.

(iv') there exists a function $g_{1} \in L_{\text {loc }}^{1}\left(\mathbb{R}^{N}\right)$ such that

$$
\left|\operatorname{div}_{x}^{a} \boldsymbol{b}(x, t)-\operatorname{div}_{x}^{a} \boldsymbol{b}(x, w)\right| \leq g_{1}(x)|t-w|
$$

for all $t, w \in \mathbb{R}$ and $x \in \mathbb{R}^{N}$

(v') the set $\mathcal{N}$, defined in (14), is a countably $\mathcal{H}^{N-1}$-rectifiable set and $\mathcal{H}^{N-1}(\mathcal{N})<$ $+\infty$;

(vi) for every $t \in \mathbb{R}$ and for $\mathcal{H}^{N-1}$ almost every $x \in \mathbb{R}^{N} \backslash \mathcal{N}$ there exists the limit (15);

(vii) for every $t \in \mathbb{R}$, the function $\boldsymbol{b}(\cdot, t)$ admits (strong) traces $\boldsymbol{b}^{ \pm}(\cdot, t)$ on $\mathcal{N}$.

As in Section 4, we shall use the notation $\beta^{ \pm}(x, t):=\left\langle\boldsymbol{b}^{ \pm}(x, t), \nu(x)\right\rangle, t \in \mathbb{R}, x \in \mathcal{N}$.

In our context, Theorem 4.7 reads as follows: For every $u \in B V\left(\mathbb{R}^{N}\right)$ the composite function $\mathbf{v}(x)=\boldsymbol{B}(x, u(x))$ belongs to $\mathcal{D} \mathcal{M}^{\infty}$ with

$$
|\operatorname{div} \mathbf{v}| \leq \sigma+M|D u|
$$

and

$$
\begin{aligned}
\widetilde{\operatorname{div}} \mathbf{v} & =\operatorname{div}_{x}^{a} \boldsymbol{B}(x, \widetilde{u}(x)) \mathcal{L}^{N}+\langle\boldsymbol{b}(x, \widetilde{u}(x)), \widetilde{D} u\rangle \\
\operatorname{div}^{j} \mathbf{v} & =\left\langle\boldsymbol{B}^{+}\left(x, u^{+}(x)\right)-\boldsymbol{B}^{-}\left(x, u^{-}(x)\right), \nu(x)\right\rangle \mathcal{H}^{N-1}\left\llcorner\left(J_{u} \cup \mathcal{N}\right) .\right.
\end{aligned}
$$

Remark 5.1. Let us point out that our hypotheses include (and actually are modeled on) the case $\boldsymbol{B}(x, u)=\widehat{\boldsymbol{B}}(k(x), u)$ where $k \in S \mathcal{D} \mathcal{M}^{\infty} \cap L^{\infty}\left(\mathbb{R}^{N} ; \mathbb{R}^{N}\right), \mathcal{H}^{N-1}\left(J_{k}\right)<+\infty$ and $\widehat{\boldsymbol{B}} \in C^{1}\left(\mathbb{R}^{N} \times \mathbb{R}, \mathbb{R}^{N}\right) \cap \operatorname{Lip}\left(\mathbb{R}^{N} \times \mathbb{R}, \mathbb{R}^{N}\right)$.

Remark 5.2. The assumption (vii) of existence of traces may appear too strong to be useful for applications. On the other hand, a situation we have in mind is the following. Let us consider the system

$$
\left\{\begin{array}{l}
\operatorname{div} \boldsymbol{A}_{1}(u)=0, \\
\operatorname{div} \boldsymbol{A}_{2}(u, v)=0,
\end{array}\right.
$$

where $u, v: \mathbb{R}^{N} \rightarrow \mathbb{R}$, and the fluxes $\boldsymbol{A}_{1}: \mathbb{R} \rightarrow \mathbb{R}^{N}, \boldsymbol{A}_{2}: \mathbb{R} \times \mathbb{R} \rightarrow \mathbb{R}^{N}$ are regular functions. In general, a solution $u$ of the first equation need not be of bounded variation. Nevertheless, if $\boldsymbol{A}_{1}$ is genuinely nonlinear, then $u$ has a quasi-BV structure, in the sense of De Lellis, Otto and Westdickenberg (see [17]). In particular, there exists a $\mathcal{H}^{N-1}$-rectifiable set $\mathcal{N}$ 
such that $u$ has left and right traces $u^{ \pm}$on $\mathcal{N}$, and it has vanishing mean oscillation at every $x \notin \mathcal{N}$. The second component $v$ is then a solution of the equation

$$
\operatorname{div} \boldsymbol{B}(x, v)=0
$$

where $\boldsymbol{B}(x, v):=\boldsymbol{A}_{2}(u(x), v)$. The vector field $\boldsymbol{B}$ admits traces on $\mathcal{N}$, given by $\boldsymbol{B}^{ \pm}(x, v)=$ $\boldsymbol{B}\left(u^{ \pm}(x), v\right), x \in \mathcal{N}, v \in \mathbb{R}$. In particular, assumption (vii) is satisfied. Unfortunately, the quasi-BV structure of $u$ is not enough to have Assumption 4.2 (vii) satisfied, since $\boldsymbol{B}(\cdot, v)$ is only of bounded mean oscillation at every point $x \notin \mathcal{N}$. Nevertheless, we think that our analysis can be a good starting point in order to obtain uniqueness results for the Cauchy problems related to the evolutionary version of the triangular system (26).

\subsection{Uniqueness of entropy solutions.}

Definition 5.3 (Convex entropy pair). We say that $(S, \boldsymbol{\eta})$ is a convex entropy pair if $S \in C^{2}(\mathbb{R})$ is a convex function, and $\boldsymbol{\eta}=\left(\eta_{1}, \ldots, \eta_{N}\right)$ is defined by

$$
\eta_{i}(x, v):=\int_{0}^{v} b_{i}(x, w) S^{\prime}(w) d w, \quad i=1, \ldots, N .
$$

In the above definition and in the sequel, $b_{i}=\boldsymbol{b} \cdot e_{i}$ are the components of $\boldsymbol{b}$.

Note that according to the previous discussion, $\boldsymbol{\eta}(\cdot, v) \in S \mathcal{D} \mathcal{M}^{\infty}$ for every $v \in \mathbb{R}$ and its divergence is given by

$$
\begin{aligned}
\operatorname{div}_{x} \boldsymbol{\eta}(\cdot, v) & =\left(\int_{0}^{v} \operatorname{div}_{x}^{a} \boldsymbol{b}(x, w) S^{\prime}(w) d w\right) \mathcal{L}^{N} \\
& +\left(\int_{0}^{v}\left(\beta^{+}(x, w)-\beta^{-}(x, w)\right) S^{\prime}(w) d w\right) \mathcal{H}^{N-1}\llcorner\mathcal{N} .
\end{aligned}
$$

Definition 5.4 (Entropy solutions). A function

$$
u \in C\left([0, T] ; L^{1}\left(\mathbb{R}^{N}\right)\right) \cap L^{\infty}\left((0, T) \times \mathbb{R}^{N}\right) \cap B V\left((0, T) \times \mathbb{R}^{N}\right)
$$

is an entropy solution of (22) if $u$ is a solution to (22) in the sense of distributions, and there exists a (everywhere defined) Borel representative $\hat{u}$ of $u$ with $|\hat{u}(t, x)| \leq\|u\|_{\infty}$ such that, for every convex entropy pair $(S, \boldsymbol{\eta})$, one has

$$
\begin{aligned}
\partial_{t} S(u) & +\operatorname{div}(\boldsymbol{\eta}(x, u)) \\
& -\left.\operatorname{div} \boldsymbol{\eta}(x, v)\right|_{v=\hat{u}(t, x)}+\left.S^{\prime}(\hat{u}) \operatorname{div} \boldsymbol{B}(x, v)\right|_{v=\hat{u}(t, x)} \leq 0
\end{aligned}
$$

in the distributional sense. Here, by $\left.\operatorname{div} \boldsymbol{B}(x, v)\right|_{v=\hat{u}(t, x)}$ we mean the measure whose action on a bounded and Borel function $\varphi=\varphi(t, x)$ is given by

$$
\begin{aligned}
\int_{0}^{T} d t \int_{\mathbb{R}^{N}} \varphi(t, x) \operatorname{div}_{x}^{a} \boldsymbol{B}(x, \hat{u}(t, x)) d x \\
\quad+\int_{0}^{T} d t \int_{\mathcal{N}} \varphi(t, x)\left\langle\boldsymbol{B}^{+}(x, \hat{u}(t, x))-\boldsymbol{B}^{-}(x, \hat{u}(t, x)), \nu(x)\right\rangle d \mathcal{H}^{N-1}(x),
\end{aligned}
$$

and the same for $\left.\operatorname{div} \boldsymbol{\eta}(x, v)\right|_{v=\hat{u}(t, x)}$.

With these definitions at hand we can now restate our main result: 
Theorem 5.5. Let $\boldsymbol{b}$ satisfy the assumptions listed at the beginning of the Section, and let $u_{1}$ and $u_{2}$ be two entropy solutions of (22), then

$$
\int_{\mathbb{R}^{N}}\left|u_{1}(T, x)-u_{2}(T, x)\right| d x \leq \int_{\mathbb{R}^{N}}\left|u_{1}(0, x)-u_{2}(0, x)\right| d x .
$$

5.2. Sketch of the proof of Theorem 5.5. The proof follows the lines of the one of Theorem 3.5 in [10]. We recall here only the main points, giving references to the details in [10]. As a first reduction, it is not restrictive to consider only non-negative solutions.

Kinetic formulation. Let us consider the measure-valued vector field

$$
\boldsymbol{a}(\cdot, v):=\left(\boldsymbol{b}(\cdot, v) \mathcal{L}_{x}^{N},-\operatorname{div}_{x} \boldsymbol{B}(\cdot, v)\right) .
$$

Note that $\boldsymbol{a}$ is a Radon measure and $\operatorname{div}_{x, v} \boldsymbol{a}=0$.

By a kinetic solution we mean a function

$$
u \in C\left([0, T] ; L^{1}\left(\mathbb{R}^{N}\right)\right) \cap L^{\infty}\left((0, T) \times \mathbb{R}^{N}\right) \cap B V\left((0, T) \times \mathbb{R}^{N}\right)
$$

which is a distributional solution of $(22)$, and satisfies the following property: there exists a (everywhere defined) Borel representative $\hat{u}$ of $u$ with $|\hat{u}(t, x)| \leq\|u\|_{\infty}$, and a positive measure $m=m(t, x, v)$ with $m\left((0, T) \times \mathbb{R}^{N+1}\right)<+\infty$, such that the function $(t, x, v) \mapsto$ $\chi(v, \hat{u}(t, x))$ satisfies

$$
\partial_{t} \chi(v, \hat{u}(t, x))+\operatorname{div}_{x, v}[\boldsymbol{a}(x, v) \chi(v, \hat{u}(t, x))]=\partial_{v} m(t, x, v)
$$

in the sense of distributions. Here the function $\chi$ is defined by

$$
\chi(v, u):= \begin{cases}1 & \text { if } v<u \\ 1 / 2 & \text { if } v=u \\ 0 & \text { if } u<v\end{cases}
$$

The first step consists in proving that $u$ is an entropy solution of (22) if and only if it is a kinetic solution.

The proof is almost the same of the one of Theorem 3.9 in [10]. We mention only that, if $u$ is an entropy solution, the kinetic measure $m$ is defined first of all as a distribution by

$$
\begin{aligned}
\langle m, \psi\rangle & =-\int_{(0, T) \times \mathbb{R}^{N+1}} d t d x d v \partial_{t} \psi(t, x, v) \int_{0}^{v} \chi(w, u(t, x)) d w \\
& -\int_{(0, T) \times \mathbb{R}^{N+1}} d t d x d v \nabla_{x} \psi(t, x, v) \int_{0}^{v} \boldsymbol{b}(x, w) \chi(w, u(t, x)) d w \\
& +\int_{(0, T) \times \mathbb{R}^{N+1}} d t d x d v \boldsymbol{B}(x, v) \chi(v, \hat{u}(t, x)) \nabla_{x} \psi(t, x, v) .
\end{aligned}
$$

Reasoning as in [10] we can prove that $m$ is a positive measure, with finite mass.

Kato contraction property. By Cavalieri's principle, it is enough to prove the following contraction property (see [10, Thm. 5.1]).

Theorem 5.6. Let $u_{1}, u_{2}$ be two entropy solution of (22), with corresponding everywhere defined Borel representatives $\hat{u}_{1}, \hat{u}_{2}$. Setting $f_{i}(t, x, v):=\chi\left(v, \hat{u}_{i}(t, x)\right), i=1,2$, we have that

$$
\int_{\mathbb{R}^{N+1}}\left|f_{1}-f_{2}\right|(T, x, v) d x d v \leq \int_{\mathbb{R}^{N+1}}\left|f_{1}-f_{2}\right|(0, x, v) d x d v .
$$

The proof of this theorem is obtained as a consequence of the following intermediate result (see [10, Prop. 5.3]). 
Proposition 5.7. Let $u_{1}, u_{2}$ be two entropy solution of (22), with corresponding representatives $\hat{u}_{1}, \hat{u}_{2}$. Setting $f_{i}(t, x, v):=\chi\left(v, \hat{u}_{i}(t, x)\right), i=1,2$, we have that

$$
\begin{aligned}
\int_{\mathbb{R}^{N+1}}\left|f_{1}-f_{2}\right|(T, x, v) d x d v & \leq \int_{\mathbb{R}^{N+1}}\left|f_{1}-f_{2}\right|(0, x, v) d x d v \\
& +\int_{0}^{T} \int_{\mathcal{N}} W\left(u_{1}, u_{2}\right) d \mathcal{H}^{N-1} d t
\end{aligned}
$$

where

$$
\begin{aligned}
W\left(u_{1}, u_{2}\right):= & \left\langle\boldsymbol{B}^{+}\left(u_{1}^{+}\right), \nu\right\rangle\left[-2 \chi\left(u_{1}^{+}, u_{2}^{+}\right)+2 \chi\left(u_{1}^{-}, u_{2}^{-}\right)\right] \\
& +\left\langle\boldsymbol{B}^{+}\left(u_{2}^{+}\right), \nu\right\rangle\left[-2 \chi\left(u_{2}^{+}, u_{1}^{+}\right)+2 \chi\left(u_{2}^{-}, u_{1}^{-}\right)\right] .
\end{aligned}
$$

The proof of this proposition is long and technical, but can be done exactly as in [10] using the new chain rule proved in Section 4.

Finally, Theorem 5.6 follows from Proposition 5.7 by proving that $W\left(u_{1}, u_{2}\right) \leq 0$ on $(0, T) \times \mathcal{N}$. This is a purely algebraic fact that follows from the entropy condition and the existence of traces, see the proof of Theorem 5.1 in [10] for details.

Acknowledgments. The authors would like to thank Guido De Philippis for some useful discussions during the preparation of the manuscript.

\section{REFERENCES}

[1] Ambrosio L., Crasta G., De Cicco V., De Philippis G., A nonautonomous chain rule formula in $W^{1, p}$ and in BV, Manuscripta Math. 140 (2013) no. 3, 461-480.

[2] Ambrosio L., Crippa G., Maniglia S., Traces and fine properties of a BD class of vector fields and applications, Ann. Fac. Sci. Toulouse Math. (6), 14 (2005), 527-561.

[3] Ambrosio L., Dal Maso G., A general chain rule for distributional derivatives, Proc. Amer. Math. Soc., 108, (1990), 691-702.

[4] Ambrosio L., De Lellis C., Malý, J., On the chain rule for the divergence of BV-like vector fields: applications, partial results, open problems, in Perspectives in nonlinear partial differential equations, Contemp. Math. 446, 31-67.

[5] Ambrosio L., Fusco N., Pallara D., Functions of bounded variation and free discontinuity problems, Oxford University Press, (2000).

[6] Anzellotti G., Pairings between measures and bounded functions and compensated compactness, Ann. Mat. Pura Appl. 185 (1983), 293-318.

[7] Chen G.-Q., Frid, H., Divergence-measure fields and hyperbolic conservation laws, Arch. Ration. Mech. Anal., 147, (1999), 89-118.

[8] Chen, G.-Q., Torres, M., Divergence-measure fields, sets of finite perimeter, and conservation laws, Arch. Ration. Mech. Anal. 175 (2005), 245-267.

[9] Crasta G., De Cicco V., A chain rule formula in BV and applications to conservation laws, SIAM J. Math. Anal., 43 (2011), no. 1, 430-456.

[10] Crasta G., De Cicco V., De Philippis G., Kinetic formulation and uniqueness for scalar conservation laws with discontinuous flux, Comm. Partial Differential Equations, 40 (2015), no. 4, 694-726.

[11] Crasta G., De Cicco V., De Philippis G., Ghiraldin F., Structure of solutions of multidimensional conservation laws with discontinuous flux and applications to uniqueness. Arch. Ration. Mech. Anal., 221, (2016), 961-985.

[12] De Cicco V., Lower semicontinuity for nonautonomous surface integrals. Rend. Lincei Mat. Appl., 26 (2015), 1-21.

[13] De Cicco V., Nonautonomous chain rules in BV with Lipschitz dependence. To appear.

[14] De Cicco V., Fusco N., Verde A., On $L^{1}$-lower semicontinuity in BV, J. of Convex Anal., 12 (2005), $173-185$.

[15] De Cicco V., Fusco N., Verde A., A chain rule formula in BV and application to lower semicontinuity, Calc. Var. Partial Differential Equations, 28 (2007), no. 4, 427-447. 
[16] De Cicco V., Leoni G., A chain rule in $L^{1}\left(\right.$ div; $\left.\mathbb{R}^{N}\right)$ and its applications to lower semicontinuity, Calc. Var. Partial Differential Equations, 19, (2004), no. 1, 23-51.

[17] C. De Lellis, F. Otto, and M. Westdickenberg, Structure of entropy solutions for multi-dimensional scalar conservation laws, Arch. Ration. Mech. Anal. 170 (2003), no. 2, 137-184.

[18] Leoni G., Morini M., Necessary and sufficient conditions for the chain rule in $W_{\text {loc }}^{1,1}\left(\mathbb{R}^{N} ; \mathbb{R}^{d}\right)$ and $B V_{\text {loc }}\left(\mathbb{R}^{N} ; \mathbb{R}^{d}\right)$, Comm. Pure Appl. Math., 58 (2005), no. 8, 1051-1076.

[19] Panov E. Yu., Existence and strong pre-compactness properties for entropy solutions of a first-order quasilinear equation with discontinuous flux, Arch. Ration. Mech. Anal. 195 (2010), no. 2, 643-673.

[20] Scheven C., Schmidt T., BV supersolutions to equations of 1-Laplace and minimal surface type, J. Differential Equations 261 (2016), 1904-1932.

[21] Vol'pert A.I., Spaces BV and quasilinear equations, Mat. Sb. (N.S.) 73 (115) (1967), 255-302.

[22] Vol'pert A.I., Hudjaev S.I., Analysis in classes of discontinuous functions and equations of mathematical physics, Mechanics: Analysis, vol. 8, Martinus Nijhoff Publishers, Dordrecht, 1985.

Dipartimento di Matematica "G. Castelnuovo", Univ. Di Roma I, P.le A. Moro 2 - I-00185 ROMA (ITALY)

E-mail address: crasta@mat.uniroma1.it

Dipartimento di Scienze di Base e Applicate per l'Ingegneria, Univ. di Roma i, Via A. SCARPA 10 - I-00185 Roma (ItAly)

E-mail address: virginia.decicco@sbai.uniroma1.it 